\title{
Avaliação da cultura de segurança do paciente em Centro Cirúrgico
}

\author{
Evaluation of patient safety culture in Surgery Center \\ Evaluación de la cultura de seguridad del paciente en Centro Quirúrgico
}

Thâmy Canova Da Correggio ${ }^{1}$, Lucia Nazareth Amante² ${ }^{2}$ Sayonara de Fátima Faria Barbosa ${ }^{3}$

RESUmo: Objetivo: Analisar a cultura de segurança em Centro Cirúrgico de um hospital universitário do sul do Brasil, sob ponto de vista dos seus profissionais. Método: Estudo exploratório, descritivo e transversal, com abordagem quantitativa, desenvolvido de julho a outubro de 2011, por meio da aplicação do Questionário de Atitudes de Segurança, a 68 profissionais da Equipe de Enfermagem e Equipe Cirúrgica. Resultados: As médias obtidas pelo instrumento, nas dimensões Clima de trabalho em equipe, Clima de segurança, Satisfação no trabalho, Reconhecimento do estresse, Percepção da gerência e Condições de trabalho foram abaixo de 75 pontos, revelando que os trabalhadores não percebem um clima de segurança para executar o seu trabalho. Conclusões: Conclui-se que as fragilidades identificadas em todas as dimensões analisadas levam os profissionais a perceber ausência de clima de segurança na realização de suas atividades. Sugere-se a promoção da educação permanente aos profissionais e a adoção de práticas seguras pela instituição, para que a segurança do paciente seja vista como prioridade.

PALAVRAS-CHAVE: Enfermagem Perioperatória. Segurança do Paciente. Atitude do Pessoal de Saúde.

ABSTRACT: Objective: To evaluate safety culture at the Surgery Center of a university hospital in southern Brazil under the point of view of its professionals. Method: An exploratory, descriptive, transverse study of qualitative approach developed between July and October 2011 by means of the application of the Safety Attitudes Questionnaire (SAQ) to 68 healthcare professionals of the surgical team. Results: The dimensions Teamwork Climate, Safety Climate, Job Satisfaction, Stress Recognition, Perceptions of Management, and Working Conditions presented averages below 75 points, which reveals that workers do not perceive a safe environment to perform their work. Conclusions: The weaknesses identified in all dimensions analyzed lead the professionals to realize the absence of safety culture in carrying out their daily activities. It is necessary to implement protocols to establish a non-punitive culture by offering in-work education for the team and getting support from the administration so that patient safety is seen as a priority by the institution.

KEYWORDS: Perioperative Nursing. Patient Safety. Healthcare Team Attitude.

RESUMEN: Objetivo: analizar la cultura de seguridad en el Centro Quirúrgico de un hospital universitario del sur de Brasil, desde el punto de vista de sus profesionales. Método: estudio exploratorio, descriptivo y transversal con enfoque cuantitativo, desarrollado entre julio y octubre de 2011, mediante la aplicación del Cuestionario de Actitudes de Seguridad, en 68 profesionales de los equipos de enfermería y quirúrgico. Resultados: las medias obtenidas por el instrumento, en las dimensiones Clima de trabajo en equipo, Clima de seguridad, Satisfacción laboral, Reconocimiento del estrés, Percepción de la gestión y Condiciones de trabajo, puntuaron por debajo de 75 puntos, revelando, así, que los trabajadores no perciben un clima seguro para ejecutar su trabajo. Conclusiones: se concluye que las debilidades identificadas, en todas las dimensiones analizadas, provocan que los profesionales no perciban un clima seguro en la realización de sus actividades. Se sugiere promocionar la educación permanente de los profesionales y que la institución adopte prácticas seguras, a fin de que la seguridad del paciente sea vista como prioritaria.

Palabras Clave: Enfermería Perioperativa. Seguridad del Paciente. Actitud del Personal de Salud.

\footnotetext{
${ }^{1}$ Enfermeira. Mestre em Saúde pelo Mestrado Profissional associado à Residência Integrada Multiprofissional em Saúde do Hospital Universitário da Universidade Federal de Santa Catarina (UFSC). Enfermeira do Hospital Nereu Ramos.

Rua Flor de Liz, 49. Bairro Bela Vista III. São José, SC, Brasil.

Telefone: (48) 8431-1086. E-mail: thamydacorreggio@gmail.com

${ }^{2}$ Enfermeira. Doutora. Professora Adjunta do Departamento de Enfermagem da UFSC no curso de Graduação em Enfermagem, no Mestrado Profissional Gestão do Cuidado em Enfermagem e no Programa de Pós-Graduação em Enfermagem. Membro do Grupo de Pesquisa Clínica, Tecnologias e Informática em Saúde e Enfermagem (GIATE).

Rua Des. Pedro Silva, 3162 apto. 210. Itaguaçu. CEP 88080-701. Florianópolis, SC, Brasil.

Telefone: (48) 3879-0242; (48) 9911-5466. E-mail: lucia.amante@ufsc.br

${ }^{3}$ Enfermeira. Doutora em Ciências. Professora Adjunta do Departamento de Enfermagem da UFSC no curso de Graduação em Enfermagem, no Mestrado Profissional Gestão do Cuidado em Enfermagem e no Programa de Pós-Graduação em Enfermagem. Vice-Líder do Grupo de Pesquisa Clínica, Tecnologias e Informática em Saúde e Enfermagem (GIATE).

Rod. Admar Gonzaga, 841 bloco B apto. 326. Itacorubi. Florianópolis, SC, Brasil

Telefone: (48) 9621-1830. E-mail: sayonara.barbosa@ufsc.br
} 


\section{Introdução}

O período perioperatório é um momento crítico, pois o paciente será submetido a um procedimento cirúrgico, expondo-se aos riscos e às suas diversas complicações, sendo necessária assistência de enfermagem e médica especializadas. As especificidades do período perioperatório foram muito discutidas ao longo do tempo; porém, a partir de 2002, também vêm preocupando a Organização PanAmericana da Saúde (OPAS) da Organização Mundial da Saúde (OMS), quando relacionadas à segurança do paciente, resultando na criação da Aliança Mundial para a Segurança do Paciente, em outubro de $2004^{1}$. A segurança do paciente é entendida como a redução, a um mínimo aceitável, do risco de dano desnecessário ao paciente, associado ao cuidado de saúde ${ }^{2}$.

Neste sentido, foram criados os Desafios Globais para a Segurança do Paciente, sendo que o segundo Desafio Global refere-se aos fundamentos e práticas da segurança cirúrgica, com o tema Cirurgias Seguras Salvam Vidas, considerada uma prioridade em saúde pública ${ }^{1}$. Ao estudarmos a segurança do paciente, nos deparamos com a questão da cultura de segurança, que é conhecida como o 'resultado das atitudes, percepções e competências, e dos padrões de comportamento e valores de um grupo, que irá determinar o compromisso, o estilo e a competência de uma organização de saúde, e de uma gestão de segurança'³.

Quando se trata do Centro Cirúrgico (CC), a equipe precisa trabalhar conjuntamente, de forma eficaz, e utilizar o que tem de melhor nos seus conhecimentos e capacidades em prol do paciente para evitar danos ${ }^{1}$. Como integrantes de um Programa de Residência Integrada Multiprofissional em Saúde de um Hospital Universitário do Sul do Brasil, convivemos diariamente com situações ocorridas no $\mathrm{CC}$, nas quais havia o risco para incidentes e eventos adversos, gerando angústia e estresse nos profissionais e comprometendo a segurança do paciente. O estresse dos profissionais, muitas vezes, dificulta a comunicação na Sala de Operação (SO), aumentando o potencial para causar danos. Por outro lado, a inexperiência e a pouca familiaridade com os equipamentos, a má comunicação entre os membros da equipe, a pressa, a desatenção e a fadiga também contribuem para a ocorrência de eventos adversos ${ }^{1}$. Esta fadiga pode estar relacionada ao duplo vínculo empregatício e, também, à carga horária demasiadamente alta no setor, para cobertura de atestados em diferentes turnos.

$\mathrm{O}$ cotidiano do $\mathrm{CC}$ requer profissionais habilitados tecnicamente, inclusive no aprimoramento da comunicação; esta, quando ineficaz, causa falhas nas relações, aumentando número de erros e, consequentemente, o estresse no setor, reduzindo a eficácia da equipe ${ }^{4}$. Além destes aspectos, neste $\mathrm{CC}$, as demais medidas para a segurança do paciente são incipientes, revelando a necessidade de que mudanças neste sentido sejam implementadas. Neste ínterim, a mudança cultural acerca da segurança do paciente é crucial para que sejam implantadas medidas eficientes de prevenção e redução dos riscos e eventos adversos ${ }^{5}$. Mostra-se necessária a construção de uma nova cultura, que entenda os valores, crenças e normas daquilo que é importante em uma instituição, e quais atitudes e comportamentos relacionados à segurança do paciente são suportados, recompensados e esperados 6 .

Partindo-se destas informações, das atualizações constantes que vimos realizando no âmbito profissional acerca da segurança do paciente e da preocupação com a cirurgia segura para os pacientes do Sistema Único de Saúde (SUS) que internam neste hospital, o presente estudo teve por objetivo avaliar a cultura de segurança no CC de um Hospital da Região Sul do Brasil, sob o ponto de vista de seus profissionais.

\section{Método}

Pesquisa de natureza quantitativa, não experimental, descritiva e transversal, desenvolvida no CC de um Hospital da Região Sul do Brasil, entre os meses de julho e outubro de 2011. Aprovada pelo Comitê de pesquisa com seres humanos da instituição, sob o número 1927/11. A equipe de trabalho do CC é formada por Médicos Cirurgiões e Anestesiologistas, Enfermeiros, Instrumentadores Cirúrgicos, Técnicos De Enfermagem, Residentes da Residência em Medicina (Cirurgia e Anestesia) e da Residência Multiprofissional (Enfermagem e Odontologia), estudantes de Graduação em Enfermagem, Medicina e Odontologia, Assistentes Administrativos e Funcionários de Serviços Gerais.

São realizadas cirurgias das especialidades: neurológica, cabeça e pescoço, plástica, vascular, torácica, odontológica, ginecológica, urológica e proctológica. É referência em Alta Complexidade em cirurgia bariátrica, by-pass endoarterial e endarterectomia, aumentando o envolvimento dos profissionais, pois compreende várias etapas críticas, sendo cada uma destas, uma oportunidade de falha e com potencial para causar dano aos pacientes. No mês de setembro de 2011, foram realizados 333 procedimentos cirúrgicos; em outubro de 2011, foram 270, e em novembro de 2011, foram 242. A demanda de pacientes com necessidade de cirurgias eletivas ou de urgência e emergência é proveniente das Unidades de Internação, da Unidade de Terapia Intensiva, do Serviço de Urgência e Emergência, e do Ambulatório.

A população estudada foi composta pela equipe de profissionais de saúde do $\mathrm{CC}$, que compreende Médicos Cirurgiões e Anestesiologistas, equipe de Enfermagem, Auxiliares de Saúde (Maqueiros e Escriturário) e Residentes de Medicina, da Odontologia e da Residência Multiprofissional, totalizando 148 sujeitos. Foram critérios de inclusão: serem profissionais da equipe do $\mathrm{CC}$ e serem estudantes da Graduação ou Pós-Graduação que estivessem no CC por, no mínimo, quatro semanas ${ }^{3}$. Foram excluídos os que estavam ausentes por estarem em período de férias, 
licenças ou outros afastamentos, durante o período de coleta de dados.

Todos os profissionais e estudantes que estavam no CC no período da coleta de dados foram considerados para seleção da amostra, não havendo separação por categoria profissional ou turno de trabalho. O tamanho da amostra, calculado por estimação de percentual - variável qualitativa, com nível de confiança de $99 \%$ - foi de 121 sujeitos $^{7}$. Da amostra, apenas 68 sujeitos devolveram o questionário, assim distribuídos: dois Enfermeiros; 19 Técnicos de Enfermagem; quatro Auxiliares de Saúde; dois Instrumentadores Cirúrgicos; 11 Anestesiologistas; dez Cirurgiões; 15 Residentes de Cirurgia; um Residente Multiprofissional, e três estudantes de Graduação, sendo que um profissional não respondeu. A técnica de seleção da amostra foi amostragem aleatória simples.

Como instrumento de pesquisa foi utilizado o Questionário de Atitudes de Segurança versão Sala de Operação (Safety Attitudes Questionaire - SAQ - OR version), desenvolvido na Universidade do Texas, que foi traduzido e adaptado, e cujo uso foi autorizado pelos autores ${ }^{3}$. O SAQ - OR é uma versão adaptada do Safety Attitudes Questionaire, cujos 58 itens são os mesmos, havendo pequena modificação para indicar a área em que foi utilizada ${ }^{3}$. O SAQ é um refinamento do Questionário de Atitudes Seguras na Unidade de Terapia Intensiva (ICUMAQ), que foi derivado de um instrumento amplamente utilizado na aviação comercial, o Questionário de Atitudes de Gestão de Voo (FMAQ) ${ }^{8,9}$.

O questionário é composto por 58 itens, distribuídos em seis dimensões, quais sejam: clima de trabalho em equipe; reconhecimento do estresse; clima de segurança; satisfação no trabalho; percepções de gestão; condições de trabalho ${ }^{3}$. Os dados de identificação contêm as informações sobre: idade; sexo; categoria profissional; tempo de experiência na instituição; carga horária semanal na instituição; turno de trabalho; formação profissional; se já havia respondido este questionário anteriormente; solicitação de sugestões para a segurança do paciente no Centro Cirúrgico. Cada um dos 58 itens das seis dimensões possibilita a resposta por meio de uma escala de cinco pontos tipo Likert: discordo totalmente, discordo parcialmente, neutro, concordo parcialmente, concordo totalmente. Assim, quanto maior o escore, mais positiva a atitude, com exceção dos itens que apresentam escore reverso, nos quais o menor escore indica uma atitude mais positiva (Questões $n^{\circ} 12$ e $n^{\circ} 26$ ). Embora o instrumento possua a avaliação de 58 itens, apenas 30 itens são considerados para análise das dimensões propostas pelo instrumento ${ }^{3}$.

Para analisar as respostas, foram efetuados dois cálculos. O primeiro cálculo foi para converter a escala de Likert e o segundo, para determinar a média das dimensões. Assim, no primeiro cálculo, os escores de cada item da escala de Likert foram convertidos para uma escala de 100 pontos, na qual um escore de 100 é considerado o desejável, assumindo, portanto, a seguinte pontuação de forma crescente: discordo totalmente $=0$; discordo parcialmente $=25$; neutro $=50$; concordo parcialmente $=75$; concordo totalmente $=100$. Com estes valores, as respostas de cada dimensão foram somadas e divididas pelo número de questões, resultando em uma variação de 0 a 100, sendo considerada uma atitude positiva quando este resultado foi igual ou maior a $75 \mathrm{em}$ $100^{3}$. Já no segundo cálculo, foram somados os valores de cada item de resposta, incluindo o escore reverso calculado e, por média simples, obteve-se a média de cada dimensão. A média do escore reverso foi calculada considerando a subtração do valor máximo de cada item de resposta do valor real da média da questão reversa.

Para a análise dos dados, foram consideradas atitudes positivas de cultura de segurança aquelas definidas como escores superiores a 75, equivalentes a 'concordo' ou 'concordo fortemente', na escala Likert.

Tratando-se de um questionário traduzido e adaptado, o SAQ foi previamente testado, por meio da aplicação a oito participantes, sendo três Residentes da Residência Integrada Multiprofissional e cinco acadêmicos de Enfermagem, todos em estágio curricular obrigatório, com mais de quatro semanas no CC. O resultado do pré-teste sinalizou que não havia necessidade de modificações.

Após o pré-teste, o projeto foi divulgado para todos os profissionais e, simultaneamente, estes foram convidados a participar. Neste momento, foram explicados os objetivos; o método de pesquisa; o termo de consentimento livre e esclarecido (TCLE). Após assinatura do TCLE, foram entregues, para o profissional que aceitou participar, o SAQ sem identificação e uma caneta. Os profissionais foram orientados a depositar os questionários respondidos em uma urna de madeira branca, fenestrada na sua parte superior e lacrada com chave, que permanecia em posse da pesquisadora. A urna estava devidamente identificada e disponibilizada na copa do CC. Os questionários eram retirados da urna com frequência diária ou semanal.

Os dados sofreram tratamento estatístico do software Microsoft ${ }^{\circledR}$ Office Excel Edição 2003, sendo utilizada análise estatística descritiva, com cálculo de frequência relativa, frequência absoluta e média. Para a análise social e demográfica dos profissionais, foram utilizadas estatísticas descritivas como frequências relativa e absoluta.

\section{Resultados}

Dos 148 profissionais, $68(64,76 \%)$ responderam ao questionário. No que diz respeito às características sociais e demográficas, a maioria dos profissionais era do sexo feminino (52\%); a maior representatividade profissional foi da Medicina, correspondendo a 36 profissionais (53\%). A média da idade foi de 40 anos, destacando-se a maioria como adulto jovem. Em relação ao tempo de trabalho na instituição, $40 \%$ trabalhavam na instituição havia menos de quatro anos. A distribuição das características sociais e demográficas dos participantes está apresentada na Tabela 1. 
Tabela 1. Características sociodemográficas dos profissionais de saúde de um centro cirúrgico. Florianópolis-SC, Brasil, 2011.

\begin{tabular}{|c|c|}
\hline Características sociodemográficas & n (\%) \\
\hline \multicolumn{2}{|l|}{ Categoria profissional $(n=68)$} \\
\hline Enfermeiro & $2(3 \%)$ \\
\hline Técnico de Enfermagem & $19(28 \%)$ \\
\hline Auxiliar de Enfermagem & $4(6 \%)$ \\
\hline Anestesiologista & $11(16 \%)$ \\
\hline Cirurgiões & $10(15 \%)$ \\
\hline Residente de Cirurgia & $15(22 \%)$ \\
\hline Residente Multiprofissional & $1(1 \%)$ \\
\hline Instrumentador Cirúrgico & $2(3 \%)$ \\
\hline Estudantes de Graduação & $3(5 \%)$ \\
\hline Não respondido & $1(1 \%)$ \\
\hline \multicolumn{2}{|l|}{$\operatorname{Sexo}(n=68)$} \\
\hline Feminino & $35(52 \%)$ \\
\hline Masculino & $32(47 \%)$ \\
\hline Não respondido & $1(1 \%)$ \\
\hline \multicolumn{2}{|l|}{ Idade $(n=68)$} \\
\hline $18-27$ & $9(13 \%)$ \\
\hline $28-37$ & $23(34 \%)$ \\
\hline $38-47$ & $14(21 \%)$ \\
\hline $48-57$ & $17(25 \%)$ \\
\hline 58 ou mais & $4(6 \%)$ \\
\hline Não respondido & $1(1 \%)$ \\
\hline \multicolumn{2}{|l|}{ Tempo de experiência na instituição $(n=68)$} \\
\hline$\leq 4$ anos & $27(40 \%)$ \\
\hline $5-9$ anos & $10(15 \%)$ \\
\hline 10-14 anos & $1(1 \%)$ \\
\hline 15-19 anos & $12(18 \%)$ \\
\hline 20 anos ou mais & $17(25 \%)$ \\
\hline Não respondido & $1(1 \%)$ \\
\hline
\end{tabular}

Fonte: Resultados da própria pesquisa.

A Figura 1 apresenta os escores médios de cada uma das seis dimensões da cultura de segurança do paciente, conforme o SAQ. Entre as seis dimensões de segurança avaliadas pelo SAQ, a atitude com média mais elevada foi 'Clima de Trabalho em Equipe' (64,33), enquanto a atitude 'Clima de Segurança' obteve a menor média $(50,66)$; neste sentido, o clima de trabalho em equipe foi a atitude mais valorizada pelos profissionais e a menos valorizada foi clima de segurança.

Na dimensão 'Clima de trabalho em equipe', foi verificado que o item com escore mais alto $(83,95)$ estava relacionado com a seguinte afirmação: Enfermeiras contribuem para que o paciente seja bem recebido na S.O.; o item com escore mais baixo foi Eu sou mais propenso a cometer erros em situações tensas ou hostis $(50,74)$. As dimensões 'Condições de trabalho' $(58,72)$ e 'Satisfação no trabalho' $(58,39)$ apresentaram médias aproximadas. Na dimensão 'Condições de trabalho', o item com escore mais alto foi Staffs/médicos atendentes fazem um bom trabalho na S.O. $(69,53)$ e o mais baixo foi Na Sala de Operação, há dificuldade de falar alto se eu perceber algum problema com o cuidado do paciente $(51,61)$. Na dimensão 'Satisfação no trabalho', o item com escore mais alto foi Gosto do meu trabalho $(92,27)$ e o mais baixo foi Eu tenho cometido erros que têm sido potenciais para dano ao paciente $(35,07)$.

No que se refere aos itens que contemplam a dimensão 'Reconhecimento do estresse', o item com o escore mais alto pertence à afirmação: Eu tenho o apoio necessário de outros profissionais para cuidar dos pacientes $(64,92)$ e o escore mais baixo: Estresses por problemas pessoais prejudicam meu desempenho $(47,42)$.

As dimensões 'Percepção da gerência' $(53,44)$ e 'Clima de segurança' $(50,66)$ obtiveram o menor escore. Na 'Percepção da gerência', o item $A$ administração do hospital apoia meu esforço diário se destacou de forma negativa dentro dessa dimensão, com escore de 36,71. Já no 'Clima de segurança', o item com maior escore pertence à afirmação: $E u$ me sentiria seguro sendo tratado aqui como paciente $(72,79) \mathrm{e}$ o item Equipamento médico na S.O. é adequado se destacou negativamente $(35,07)$.

\section{Discussão}

Os resultados do estudo evidenciaram que aspectos relevantes da cultura da segurança do paciente no $\mathrm{CC}$ precisam ser aprimorados, uma vez que a avaliação da média dos escores confirma que todas as seis dimensões obtiveram valores inferiores a 75 , sendo este considerado como mínimo positivo para a cultura de segurança.

Há dimensões que evidenciam a cultura de segurança do paciente neste $\mathrm{CC}$, sendo que as mais valorizadas pelos profissionais, são: 'Clima de Trabalho em Equipe', 'Condições de Trabalho' e 'Satisfação no Trabalho', obedecendo a uma ordem decrescente de valor; a menos relevante é 'Clima de Segurança'. Estes resultados assemelham-se a um estudo brasileiro $^{10}$, no qual o questionário aplicado à Equipe de Enfermagem da Estratégia de Saúde da Família e aos Agentes de Saúde do Programa de Agentes Comunitários revelou que as dimensões 'Condições de Trabalho' e 'Clima de Trabalho em Equipe' estão entre aquelas que evidenciam a cultura de segurança no âmbito daquele estudo.

Os resultados alcançados na dimensão 'Clima de Trabalho em Equipe' alertam para o fato de os participantes acreditarem que os Enfermeiros contribuem para que o paciente seja bem recebido na SO, confirmando a valorização do cuidado do Enfermeiro, que é essencial para garantia da qualidade da assistência. Esta valorização do trabalho por 


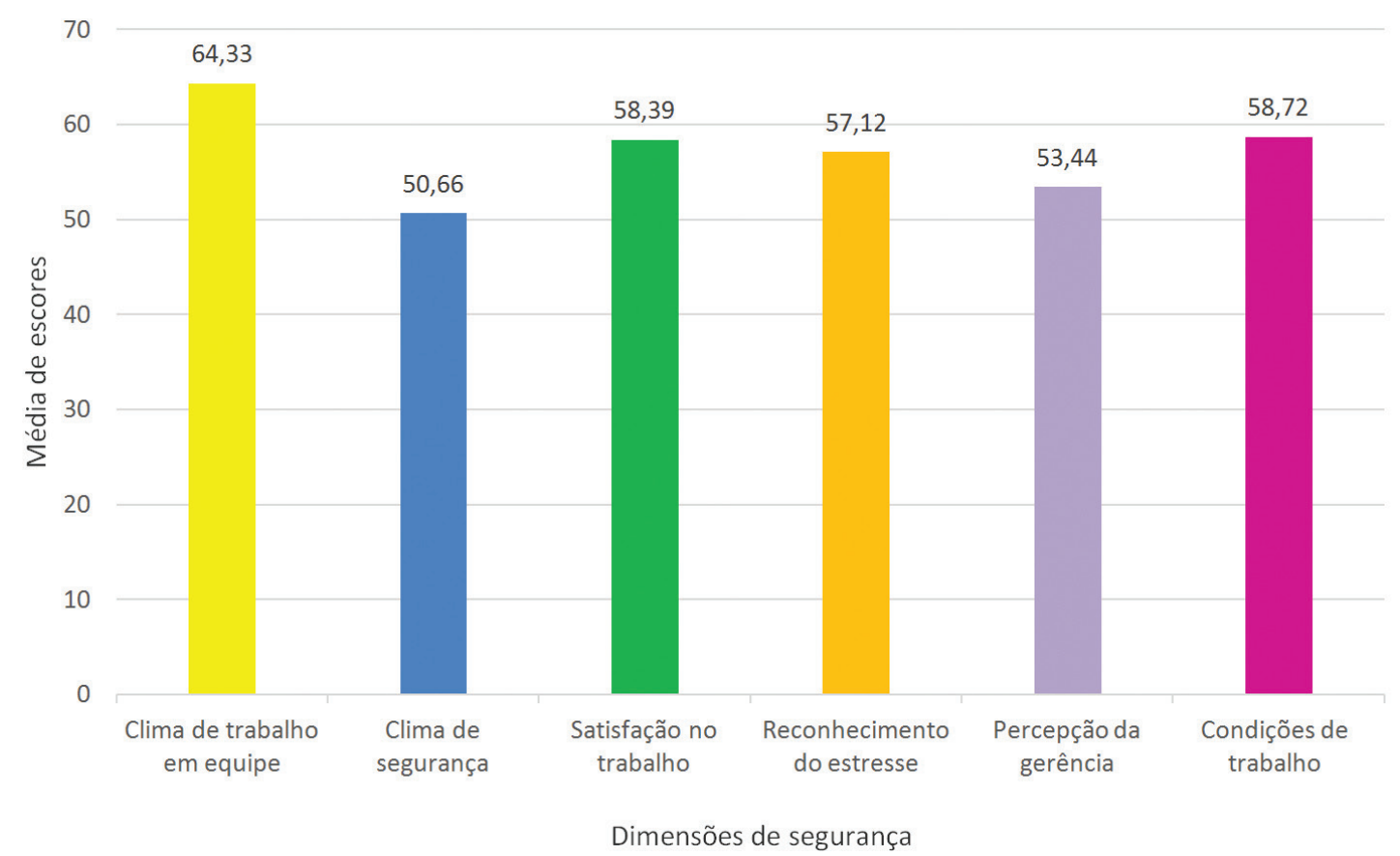

Figura 1. Média de escores obtidos a partir da avaliação da equipe de saúde que atua no Centro Cirúrgico do estudo, segundo as dimensões do questionário $\mathrm{SAQ}$ - OR traduzido e adaptado ( $\mathrm{n}=68$ ). Florianópolis-SC, Brasil, 2011. Fonte: Resultados da própria pesquisa.

outros profissionais e o reconhecimento das habilidades e potencialidades do Enfermeiro pela equipe do CC favorecem a criação de um clima de trabalho positivo, que depende de relações interpessoais harmoniosas, respeito às diferentes opiniões, desenvolvimento de confiança e de trabalho coletivo para construir um ambiente focado na segurança do paciente. Um estudo ${ }^{11}$ concluiu que o trabalho em equipe resulta em maior produtividade, considerada essencial para o C.C, além de melhorar a autoestima, o bem-estar psicológico e o apoio social, que estão fortemente relacionados à satisfação no trabalho, conduzindo a mudanças na melhoria da segurança dos pacientes.

Entretanto, nesta mesma dimensão, a metade dos participantes não percebe de maneira efetiva a propensão para erros em situações tensas ou hostis, item considerado negativo para a segurança, pois os participantes não percebem o quanto os erros intermediam nestas situações e favorecem a ocorrência de eventos adversos para os pacientes, com resultado transitório ou permanente.

Na avaliação das condições de trabalho, observou-se que os participantes consideram bom o trabalho realizado pelos médicos na $\mathrm{SO}$, em contraponto à dificuldade sentida por metade do grupo para expressar problemas relacionados ao cuidado do paciente, pressupondo uma dificuldade de abertura por parte da equipe para a discussão dos erros por eles mesmos visualizados. Há estudos brasileiros ${ }^{10,12}$ que também desvendaram esta dificuldade, corroborando que os profissionais ainda são receosos nos relatos destes problemas.

Este receio dos profissionais revela a ausência ou a dificuldade de criar um clima de segurança, evidenciado neste estudo com a menor média entre as dimensões da cultura de segurança do paciente. Esse resultado difere daquele estudo brasileiro ${ }^{10}$, no qual a dimensão 'Clima de Segurança' obteve a média mais alta. Nesta dimensão, há um contraponto: os participantes referem que se sentiriam seguros sendo tratados naquela instituição como pacientes, ao mesmo tempo em que revelam que os equipamentos médicos não são adequados na SO. Neste sentido, pode-se inferir que os profissionais não reconhecem os equipamentos médicos como potenciais facilitadores para ocorrência de incidentes, erros com consequente evento adverso, centrando toda responsabilidade pela qualidade e pela segurança do trabalho nos profissionais. Assim, entende-se que os materiais e equipamentos específicos necessários ao ato cirúrgico precisam ser previstos pelo Enfermeiro da unidade, para que o fluxo de trabalho se desenvolva harmonicamente e para que o paciente não sofra danos causados pela ausência do material, assim como pela suspensão de cirurgias. Além da presença destes na unidade, é necessário testá-los antes do procedimento cirúrgico e realizar manutenção preventiva e reparadora sempre que for necessário, conforme orientações da $\mathrm{OMS}^{(1)}$. Nesta instituição, a manutenção preventiva dos equipamentos não é realizada, fato que não contribui para a segurança, pois, a qualquer momento, pode haver uma falha no equipamento que poderia ser evitada. Confirma-se, desta forma, a não percepção dos profissionais da relação entre equipamentos e segurança.

No entanto, a satisfação no trabalho foi avaliada como a terceira atitude mais relevante pelos participantes. Destaca-se que os profissionais se orgulham em atuar na instituição 
quando referem gostar do seu trabalho e que esta instituição é um bom local de trabalho. Estes resultados confluem com outros estudos ${ }^{12,13}$, nos quais o item 'Satisfação no trabalho' obteve o escore mais alto. Alguns autores ${ }^{14,15}$ revelam que há uma ligação entre a satisfação no trabalho positiva com o aumento no desempenho e na qualidade da segurança do paciente, favorecendo maior apoio na criação de uma cultura de segurança.

O 'Reconhecimento do Estresse' foi reconhecido pela metade dos participantes como influência negativa no trabalho, com probabilidade de comprometimento na segurança do paciente, enquanto a outra metade dos participantes não tem essa percepção. Este resultado pode indicar que metade dos profissionais não reconhece a situação estressante como algo a ser revertido positivamente, mas a entendem como pertinente ao ambiente de trabalho, como situação normal para o exercício de suas funções.

Existem fatores, referentes aos recursos humanos, que interferem no desenvolvimento de um cuidado seguro e que estão intimamente ligados ao reconhecimento do estresse, como a fadiga dos profissionais pela carga horária excessiva de trabalho, a escassez de recursos humanos, as barreiras na comunicação entre os profissionais, as relações interpessoais não efetivas, as distrações, as interrupções, os erros de julgamento, a falta de atenção e o fator emocional dos profissionais; note-se que esses fatores também estão presentes no CC pesquisado ${ }^{16-19}$. Além destes fatores, a sobrecarga de trabalho, o acúmulo de tarefas rotineiras, os problemas com materiais e equipamentos que precisam de resolução, a realização de escalas de serviço e a ausência de profissionais - por férias, licenças e outros afastamentos - aumentam a fadiga e o estresse nos Enfermeiros, dificultando o desenvolvimento de ações que promovam a segurança do paciente e um cuidado especializado e centrado na proteção da vida ${ }^{3,16}$.

Os fatores estressantes identificados neste estudo, apoiados pela literatura, indicam a fragilidade da gestão hospitalar e vão ao encontro do escore significativamente baixo para a 'Percepção da Gerência'. Este resultado é consistente com outros estudos brasileiros ${ }^{12,13}$ que também identificaram que os participantes não se sentem apoiados pela gestão hospitalar e não são incluídos nas tomadas de decisão, configurando uma fragilidade na comunicação entre os gestores e os profissionais das equipes de trabalho assistencial.

\section{Conclusão}

A utilização do SAQ - OR permitiu estabelecer um panorama da segurança do paciente na visão dos profissionais do $\mathrm{CC}$, embora se ressalte a necessidade de que este instrumento seja adaptado e validado considerando a cultura brasileira, haja vista que sua concepção e validação se deram em países culturalmente diferentes do Brasil.
O resultado da avaliação da cultura de segurança no CC revelou fragilidade em todas as dimensões avaliadas. Estas mudanças requerem esforços de toda a organização hospitalar para incentivar a atenção dos profissionais na condução das ações, para o conhecimento das causas dos erros e suas formas de julgamento, através de atitudes questionadoras e prudentes, fortalecendo a cultura não punitiva e afastando o foco da atenção de quem realizou o erro.

É necessário que haja um movimento em prol da cultura de segurança no CC desta instituição pautado no modelo mental sistêmico, que entende a condição humana para o erro, admitindo que erros aconteçam e que existe a necessidade da criação de sistemas para antecipar e prevenir os erros, e interceptar os danos decorrentes deste erro. Desta forma, é favorecida a cada profissional a possibilidade de mudar sua atitude e voltar-se para o novo paradigma da cultura de segurança no seu local de trabalho, com as lideranças priorizando a adoção de sistemas que garantam a segurança do paciente. Para isto, a comissão de educação em serviço deve abordar este tema para todos os profissionais, com a promoção de capacitações regulares. Os gestores devem promover e apoiar a implementação de práticas seguras baseadas em evidências e de protocolos de atendimento.

Recomenda-se que novos estudos sejam realizados com o intuito de promover a segurança do paciente, com o entendimento de que todos são parte do sistema e que a implementação de padronizações e o melhoramento do trabalho em equipe, da comunicação e do aprendizado com erros passados são essenciais para o ambiente seguro.

\section{Referências}

1. Organização Mundial da Saúde - OMS. Segundo desafio global para a segurança do paciente: cirurgias seguras salvam vidas (orientações para cirurgia segura da OMS). Rio de Janeiro: Organização Pan-Americana da Saúde, Ministério da Saúde, Agência Nacional de Vigilância Sanitária; 2009.

2. World Health Organization - WHO. The conceptual framework for the International Classification for Patient Safety. Version 1.1. Final Technical Report. WHO; 2009 [acesso em 2011 Abr 10]. Disponível em: www.who.int/en/

3. Sexton JB, Helmreich RL, Neilands TB, Rowan K, Vella K, Boyden J. The Safety Attitudes Questionnaire: psychometric properties, benchmarking data, and emerging research. BMC Health Serv Res. 2006;6(1):44. PMid:16584553 PMCid:PMC1481614. http:// dx.doi.org/10.1186/1472-6963-6-44

4. Pereira MCMAP. Dinâmicas e percepções sobre trabalho de equipa: um estudo em ambiente cirúrgico [dissertação]. Covilhã: Faculdade de Ciências da Saúde, Universidade da Beira Interior; 2010.

5. Silva LFN. Reorientação do gerenciamento de risco hospitalar do Instituto Nacional de Traumatologia e Ortopedia [dissertação]. Rio de Janeiro: Escola Nacional de Saúde Pública Sérgio Arouca, Fundação Oswaldo Cruz; 2009.

6. Agency for Healthcare Research and Quality - AHRQ. Hospital survey on patient safety culture (Comparative Database Report 090030). AHRQ; 2009. 
7. Nassar SM, Wronscki VR, Ohira M, Wilges B, Mateus GP, Tenorio MB. SEstatNet: Sistema Especialista para o Ensino de Estatística na Web. Florianópolis: UFSC; [acesso em 2013 Out 10]. Disponível em: http://www.sestatnet.ufsc.br/

8. Merritt AC. National culture and work attitudes in commercial aviation: a cross-cultural investigation [tese]. Austin: The University of Texas; 1996.

9. Helmreich RL, Merritt AC, Sherman PJ, Gregorich SE, Wiener EL. The Flight Management Attitudes Questionnaire (FMAQ): NASA/UT/FAA (Technical Report 93-4). Austin: The University of Texas; 1993.

10. Paese F. Cultura da segurança do paciente na atenção primária à saúde [dissertação]. Florianópolis: Programa de Pós-Graduação em Enfermagem, Universidade Federal de Santa Catarina; 2010.

11. Walshe K, Boaden R. Patient Safety research into practice. New York: Open University Press; 2006.

12. Fidelis RE. Cultura de segurança: perspectiva da equipe de enfermagem em unidade de emergência adulto [dissertação]. Florianópolis: Universidade Federal de Santa Catarina; 2011.

13. Marinho MM. Cuidado de enfermagem e a cultura de segurança do paciente: um estudo avaliativo em unidades de internação cirúrgica [dissertação]. Florianópolis: Programa de Pós-Graduação em Enfermagem, Universidade Federal de Santa Catarina; 2012.
14. Wisniewski AM, Erdley WS, Singh R, Servoss TJ, Naughton BJ, Singh G. Assessment of safety attitudes in a skilled nursing facility. Geriatric Nurs. 2007;28(2):126-36. PMid:17430747. http://dx.doi. org/10.1016/j.gerinurse.2007.01.001

15. Zakari NMA. Attitude of academic ambulatory nurses toward Patient Safety Culture in Saudi Arabia. Life Science J. 2011;8(3):230-7.

16. Silva BMS. Jornada de trabalho: fator que interfere na qualidade da assistência de enfermagem. Texto \& Contexto Enferm. 2006;15(3):442-8. http://dx.doi.org/10.1590/ S0104-07072006000300008

17. Silva DC, Alvim NAT. Ambiente do Centro Cirúrgico e os elementos que o integram: implicações para os cuidados de enfermagem. Rev Bras Enferm. 2010;63(3):427-34. http://dx.doi. org/10.1590/S0034-71672010000300013

18. Pereira BMT, Pereira AMT, Correia CS, Marttos AC Jr, Fiorelli RKA, Fraga GP. Interrupções e distrações na sala de cirurgia do trauma: entendendo a ameaça do erro humano. Rev Col Bras Cir [Internet]. 2011 [acesso em 2012 Jan 7];38(5). Disponível em: http://www.scielo.br/scielo. php?pid=S0100-69912011000500002\&script=sci_arttext.

19. The Joint Commission. Health care worker fatigue and patient safety. Sentinel Event Alert [Internet]. 2011 [acesso em 2012 Jan 9];48. Disponível em: http://www.jointcommission.org/ sea_issue_48/. 